Revue d'études américaines. American Studies Journal

\title{
Entretien avec Laurent Bécue-Renard, réalisateur de of Men and War
}

\section{Géraldine Chouard}

\section{(2) OpenEdition}

\section{Journals}

Édition électronique

URL : https://journals.openedition.org/transatlantica/7252

DOI : $10.4000 /$ transatlantica. 7252

ISSN : 1765-2766

Éditeur

Association française d'Etudes Américaines (AFEA)

Référence électronique

Géraldine Chouard, «Entretien avec Laurent Bécue-Renard, réalisateur de Of Men and War »,

Transatlantica [En ligne], 2 | 2014, mis en ligne le 11 mars 2015, consulté le 05 février 2023. URL:

http://journals.openedition.org/transatlantica/7252 ; DOI : https://doi.org/10.4000/transatlantica 7252

Ce document a été généré automatiquement le 5 février 2023

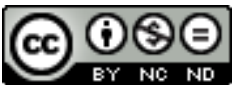

Creative Commons - Attribution - Pas d'Utilisation Commerciale - Pas de Modification 4.0 International - CC BY-NC-ND 4.0

https://creativecommons.org/licenses/by-nc-nd/4.0/ 


\title{
Entretien avec Laurent Bécue- Renard, réalisateur de of Men and War
}

\author{
Géraldine Chouard
}

Of Men and War (2014, 2h 22, Alice Films, réalisé à Paris le 19 décembre 2014) a reçu le Prix du meilleur documentaire dans la catégorie long-métrage de l'IDFA (International documentary film festival d'Amsterdam) en décembre 2014.

Géraldine Chouard: Après De guerre lasses (2002), qui portait sur les veuves de guerre bosniaques, votre dernier film, Of Men and War (2014) traite à nouveau des effets de la guerre. Est-ce une manière d'aborder la question du point de vue des hommes qui l'ont faite, après avoir observé les femmes qui l'avaient subie?

Laurent Bécue-Renard : Oui exactement. De guerre lasses était un portrait de la femme, un portrait de la mère et un portrait de la veuve. Il se trouve que le film a été tourné en Bosnie-Herzégovine, mais c'était une représentation de type mythique, beaucoup plus que circonstancielle. Ayant travaillé cette question, j'ai éprouvé le besoin, après avoir montré le film à des milliers de spectateurs en France et à l'étranger, de faire le portrait de l'homme qui n'était pas présent dans De guerre lasses. Of Men and War est donc une représentation de l'homme, du père et du guerrier. Et dans les deux cas, ces représentations visent à mettre en évidence combien la guerre perdure après la fin de la guerre, habite les protagonistes, civils ou des militaires - pour moi, ce sont toujours des civils même quand ils ont l'habit militaire - et comment elle se transmet d'une génération à l'autre, devient le patrimoine de la famille.

GC : Pourquoi avez-vous décidé d'aller tourner aux États-Unis ? Et comment s'est passé le tournage sur place pour le réalisateur français que vous étiez?

LBR : Il y a à la fois des raisons objectives et des raisons plus subjectives. La première raison objective est qu'au moment où j'ai eu l'idée du film, fin 2003 début 2004, c'était l'Amérique qui était massivement en guerre, en Afghanistan depuis deux ans et en Iraq depuis quelques mois. La deuxième est que, singulièrement, depuis la guerre du Vietnam, l'Amérique a fait un gros travail de recherche sur le traumatisme de guerre 
et de PTSD [Post-Traumatic Stress Disorder]. Des premiers travaux avaient été faits après la Première Guerre mondiale, puis après la seconde, mais c'est vraiment le Vietnam qui a ouvert un champ assez vaste de recherche dont on utilise les résultats également dans le civil, par exemple lors de catastrophes naturelles ou d'attentats. La réflexion sur le traumatisme a été très nourrie de ce travail. Ce sont donc les raisons objectives.

Il y a eu aussi des raisons beaucoup plus subjectives. En tant que cinéaste, l'idée ne me déplaisait pas d'aller à la source des représentations guerrières qu'on a tous, plus ou moins inconsciemment, venues pour l'essentiel du cinéma américain, et de les déconstruire. Les images que nous avons des guerriers nous viennent des films purement épiques sur la Seconde Guerre mondiale, ce qui a commencé avec tous les grands films des années 50 comme Le jour le plus long [The Longest Day, 1962], Les canons de Navaronne [The Guns of Navarone, 1961], jusqu'à Saving Private Ryan [1998] ou Fury [2014] qui est sorti le même jour que of Men and War, mais aussi de nombreux films anti-guerre, post-Vietnam, comme Apocalypse Now [1979], Voyage au bout de l'enfer [The Deer Hunter, 1978]. Tous ces films proposent des visions de la guerre qui, de mon point de vue, sont extrêmement éloignées de la vérité psychique des guerriers. Et donc, l'un des moteurs de mon désir d'aller filmer aux États-Unis était d'aller montrer l'envers du décor.

Après, il y a la question de la distance. Ça fait presque vingt ans que j'introduis la caméra en thérapie et j'ai cette intime conviction que c'est parce que je suis cet étranger absolu qui vient de très loin, qui appartient à une culture dont on ne connaît pas grand chose, qui passe si longtemps loin de chez lui, qui semble même avoir un temps infini, cet étranger qui n'a pas d' "agenda » de questions comme on dit en anglais, mais qui est à l'affût des questions que les protagonistes se posent à euxmêmes, c'est parce qu'il est cet étranger-là que sa présence peut être acceptée en thérapie, parce que du coup, elle reconnaît et valide leurs traumatismes. Le fait d'être ce Français en Amérique a contribué à cette légitimité. Inversement, il m'est plus aussi facile de travailler avec des gens qui me sont étrangers parce que je ne suis pas parasité par l'inconscient du langage. Pour prendre un exemple, ces garçons jurent un mot sur deux, et j'entends les jurons, mais je ne les entends pas comme tels, j'entends ce qu'ils sont en train de dire entre les jurons. Et je construis le récit sur ce qu'ils disent, pas sur les jurons. Si c'étaient des Français, peut-être que j'arriverais assez vite à un certain niveau de saturation de ces jurons. Et donc la distance qui, paradoxalement, permet la proximité existe dans les deux sens, et pour eux, et pour moi. Et c'est là aussi ce qui détermine l'Amérique. Et je pense qu'il faut aussi s'embarquer dans un voyage lointain, à la fois géographiquement et physiquement, pour suivre leur périple psychique, ce qui était aussi le cas dans De guerre lasses.

GC : A propos du proche et du lointain, vous avez fait le lien entre ce film et votre histoire familiale. Vous avez d'ailleurs dédié votre film à vos deux grands-pères dont vous montrez les portraits à la fin. En France, l'expérience de la guerre, de la première comme de la deuxième, n'est guère un sujet de discussion entre les générations, et donc on s'interroge sur le type de parole que vous avez pu trouver auprès ces jeunes vétérans américains.

LBR : Quand j'ai commencé à voir dans la presse américaine les portraits des soldats qui rentraient, plus ou moins blessés physiquement et psychiquement, et qui avaient exactement l'âge que mes deux grands-pères avaient en rentrant de la Première Guerre mondiale, quelque chose de l'ordre du déclic s'est produit. Je me suis dit que 
c'était ces hommes-là qui allaient parler à la place de mes grands-pères qui, effectivement, étant revenus dans leur vingtaine, avaient fondé leur famille après la guerre, n'en ont jamais parlé à leurs épouses, puis jamais à leurs enfants, et pour celui des deux qui a connu ses premiers petits-enfants, ne leur en a jamais parlé non plus. Et dans mon cas, ils parlaient encore moins puisqu'ils étaient déjà morts quand je suis né. Mais l'ombre de ce silence s'est portée sur la période de formation, mon enfance, mon adolescence. Évidemment, il a fallu que moi-même je m'expose à la guerre en Bosnie pendant environ un an et que je fasse ensuite le premier film pour comprendre à quel point j'étais habité par ce qui n'avait pas été exprimé. Encore une fois, l'expérience de ma famille est banale dans le contexte français. Elle était singulière pour les protagonistes, mes deux grands-pères, mais c'était une expérience qu'ont vécu toutes les familles de cette époque. Chez moi, en revanche, quelque chose ne passait pas et j'étais habité pendant toute la durée du travail sur ce film par le fait que c'était là ma quête. J'allais chercher la parole qui n'avait pas été dite. Et je le disais aux garçons, là-bas. Je leur montrais les deux photos de mes grands-pères pendant la Première Guerre mondiale.

\section{GC : Qu'est-ce que vous en disiez?}

LBR : J'en disais : "Vous voyez, ils avaient votre âge et n'ont pas parlé. Et à la troisième génération, la mienne, il se trouve que ça ne passe pas ». Évidemment, c'était un peu vertigineux parce que, moi, j'avais l'âge d'être le père de ces garçons et je leur disais que j'étais leur petit-fils, mais ils comprenaient parfaitement qu' à la troisième génération quelqu'un vienne leur dire le résultat du silence, qui peut devenir obsessionnel. Ils le comprenaient d'autant mieux que ceux qui ont des enfants, on les voit dans le film, sont en pleine conscience qu'ils sont en train de passer à la génération suivante les effets du traumatisme. Une partie du lien avec ces garçons et de la légitimité de la place de la caméra en thérapie vient également du fait qu'il y a une quête qui peut faire sens pour tout le monde. Cette quête existait déjà dans De guerre lasses. Je n'étais pas parti en Bosnie comme cinéaste, mais dans mes dernières semaines de mon séjour de guerre à Sarajevo, j'ai rencontré cette thérapeute bosniaque qui est le pivot du film et le pendant de Fred Gusman dans of Men and War. Je lui ai tout de suite dit que j'étais intrigué par ce qu'elle faisait et lui ai demandé si je pouvais assister à une séance de thérapie collective. Elle a accepté et là, sur le champ, j'ai eu une espèce de révélation : j'étais sidéré de voir ces femmes en situation de commencer à verbaliser ce qu'elles avaient éprouvé pendant la guerre. C'était tellement contraire à tout ce silence qui avait entouré ma vie familiale, y compris mes parents qui étaient de jeunes adultes civils pendant la Seconde Guerre mondiale. Et à l'époque je grandissais, dans les années 1960, il me semblait encore que rien de ce qui avait éprouvé de manière sensible ne devait être exprimé.

GC: Est-ce qu'on parle de «syndrome de stress post-traumatique dans la culture française »? Le phénomène semble mieux identifié, plus reconnu aux Etats-Unis.

LBR : Aux États-Unis, que ce soit dans la presse écrite ou audiovisuelle, c'est un terme qui est employé très régulièrement. Je ne suis pas convaincu que le grand public ait une idée précise de ce que cela représente, mais au moins, ils savent qu'il y a là quelque chose qui appartient aux soldats qui rentrent et que leur vie n'est pas si simple. Surtout pour ceux qui ne sont pas blessés physiquement, ceux dont l'apparence apparaît tout à fait « ordinaire ». 
GC: Ce qui est aussi frappant dans votre film, c'est que ces hommes que vous filmez semblent pour la plupart avoir relativement peu de mots, mais ils sont sans arrêt en quête du mot juste. Tout ce qu'ils disent est ponctué de jurons, mais ils donnent l'impression de vraiment chercher à aller au plus près de leur expérience traumatisante.

LBR : Oui, c'est une des choses les plus bouleversantes. C'est à la fois la recherche constante de l'expression de leur expérience, comme vous dîtes, qu'on voit aussi quand ils essaient de créer des représentations physiques en faisant des maquettes ou en créant un champ de bataille en pâte à modeler, ce que j'interprète, à tort ou à raison, comme la recherche d'un moyen d'expression, pour dire quelque chose. Ce qui est très impressionnant, c'est à quel point, dès qu'ils sont en situation thérapeutique, même avec ce que vous appelez une éventuelle «pauvreté du langage ", leur discours est articulé et ils sont capables de nommer les choses. Alors, ces choses qu'ils nomment, c'est peut-être juste le contour de la «béance » qui est évoquée à un moment donné dans le film, mais c'est un contour assez précis. Je pense que le travail thérapeutique permet de nommer vraiment le bord du précipice, qui, en soi, et pour tout le monde, est innommable.

\section{GC : Cet effort est tout à fait visible en effet, très poignant.}

LBR : Vous avez raison d'insister là-dessus parce qu'ils jouent le jeu. Ils veulent être des bons patients.

GC : Déjà, ils sont là.

LBR : Oui, ils sont là. Ils résistent parfois, mais en même temps, ils jouent le jeu.

GC: On est frappé de voir à quel point l'expression de leur traumatisme est décontextualisée de tout enjeu politique. Est-ce que c'est de votre fait, au montage, ou est-ce qu'ils ont vraiment évacué cette question?

LBR : Avant d'arriver en thérapie, il s'est passé en moyenne deux, trois ans. Une période qui a été pour eux une chute dans un précipice sans fond: désocialisation, éclatement des familles, abus en tous genres (alcool, drogue, violence familiale, violence de la société, violence de la rue), tentative de suicide, de la prison parfois, comme on le dit en anglais, you name it. Et puis dans cette chute, à un moment donné, ils ont eu « la chance » d'avoir quelqu'un qui les aimait suffisamment pour leur dire «tout ça va très mal se terminer, pour toi, pour nous qui t'aimons, il faut que tu fasses quelque chose " et qui leur ont trouvé cet établissement. Ces personnes leur ont peut-être parfois fait des pressions, du chantage moral, en leur disant «si tu n'y vas pas, moi, je sors de ta vie » ou « je ne m'occupe plus de toi ». C'est possible, mais ils y ont quand même été de manière volontaire. Pendant les cinq mois qu'on passe dans le centre et pendant lequel on les suit à l'écran, il n'y a pas une seconde où une bataille extrêmement violente ne fait pas rage en eux, entre leur pulsion mortifère et le désir de vivre, propre à l'espèce, mais eux sont au bord du précipice tout le temps. Ils l'étaient pendant les trois ans qui les ont amenés à entrer en thérapie et ils le sont toujours, pendant tout le film. Tout cela pour dire que la question politique n'est pas $\mathrm{du}$ tout, du tout, du tout celle du moment. Ce qui ne veut pas dire qu'elle surgira ou qu'elle ne surgira pas plus tard, mais là, il s'agit de savoir si on va survivre. Et ce n'est pas gagné. D'ailleurs vous voyez bien qu'on va en perdre un, un an après la thérapie. Et on aurait pu les perdre tous, tout aussi bien. L'entreprise de thérapie dont il est question cherche à trouver un premier point d'ancrage, sachant que ce sera le travail de toute une vie. Et c'est ce que fait Fred Gusman. Il cherche à les réancrer dans la vie. 
Maintenant, en tant que témoin de ce cheminement, je voudrais dire que si ces garçons avaient eu un discours politique, d'abord, il aurait sans doute été stéréotypé et très peu incarné. Et puis, je pense que ça aurait été une fuite, et ça m'aurait beaucoup inquiété. Imaginez-les en train de dire «c'est la faute à » - à tel gouvernement, tel président, même la faute à leur père. Ceci aurait voulu dire, de mon point de vue, qu'ils n'affrontaient pas la façon dont ils avaient été blessés dans leur humanité propre.

GC : Oui, mais il y a parfois plusieurs vérités qui existent en même temps. Dans leurs accès de colère, on imagine qu'une accusation politique aurait pu soudain sortir.

LBR : Mais ce qui sort vraiment, c'est leur combat pour la survie. J'ai entendu des gens qui avaient vu De guerre lasses me dire que les femmes n'étaient pas très politisées, et qu'ils s'attendaient à ce que ces femmes, qui pour certaines d'entre elles, avaient perdu vingt, trente, quarante, hommes de leur famille, aient un discours politique sur l'ennemi. D'abord, là aussi, il aurait été très stéréotypé : l'ennemi est affreux. Bon, mais la cause est entendue. Et elles n'auraient pas été en train d'affronter la manière dont cette catastrophe les a affectées. Les femmes de Bosnie cherchaient aussi avec leur thérapeute un point d'ancrage où continuer à vivre a encore un peu de sens. Je crois que c'est l'enjeu véritable. Là encore, je me méfierais beaucoup des patients qui trouveraient du sens uniquement dans l'engagement politique.

GC : On les voit tout de même défiler patriotiquement, un 4 juillet. Ils font partie de la parade locale [Yountville, CA].

LBR: Regardez bien. Ils ne sont pas en uniforme. Ils portent un T-shirt de l'institution, Pathway. Ils défilent ès-qualité de patients. C'est important. Ils se rendent compte que ces civils qui les applaudissent n'ont aucune idée de ce qu'ils ont vécu. Cette scène parle de l'incompréhension totale entre le front et l'arrière. Quelque soit l'honnêteté et la générosité de l'arrière, de ces gens qui les remercient de tout ce qu'ils ont fait, ils ne se rendent absolument pas compte du coût humain que cette expérience représente pour chacun.

GC : C'est en effet visible dans le regard de certains des hommes.

LBR : Oui. Eux se rendent compte de cette incompréhension, mais les gens dans la rue, non. Et une fois la parade terminée, ils retournent en thérapie, parce que la vérité est là, dans ce qui se dit en thérapie, pas dans la parade. Effectivement, ils agitent le drapeau, mais le cœur n'y est pas trop. Ce n'est pas qu'ils rejettent le drapeau, c'est juste que l'irréconciabilié est complète.

GC : Pouvez-vous nous en dire davantage sur Fred Gusman, le thérapeute. On s'interroge en effet, en tant que spectateur, sur son propre parcours. II a la carrure de ces hommes, il sait leur parler, il sait les remettre en place et les reconduire sur un chemin, qui est d'ailleurs le nom de l'institution. Comment est-il arrivé là?

LBR : Il est l'un des leurs, au sens où il a été soldat, mobilisé pour la guerre au Vietnam. Il a observé chez son propre frère, qui était parti en même temps que lui, un très grand traumatisme de guerre. Ils avaient un père qui avait fait la Seconde Guerre mondiale et qui était d'une extrême violence, ce qu'il attribuait au traumatisme de guerre. Je crois qu'en rentrant il a eu une sorte de révélation : il voulait consacrer sa vie à soigner ses frères d'armes. Il a donc a fait des études pour devenir thérapeute, et puis, assez jeune homme, il a contacté le Veterans Affairs, qui est quand même, il faut le rappeler, un état dans l'état: 200.000 fonctionnaires, 100 milliards de dollars de budget, le premier système de santé aux États-Unis pour 24 millions d'anciens 
combattants. Il leur a proposé de créer un centre thérapeutique résidentiel pour accueillir tous ces vétérans du Vietnam qu'on voyait dans les rues des grandes villes américaines à la fin des années 70, ceux qu'on voit aussi dans les films américains de l'époque, comme Taxi Driver [1976]. Sa proposition a tout de suite été acceptée et d'un coup, il a eu cent lits sous sa responsabilité, dont il s'en est occupé pendant vingt-cinq ans, un peu plus même. C'est en cette capacité-là que je l'ai connu, il y a dix ans. Il travaillait toujours. Il y a eu jusqu'à sept établissements sur le territoire fédéral, regroupé sous l'égide du « National Center for PTSD ». A l'époque où je l'ai connu, il regrettait que l'administration ne créât pas de centre dédié aux jeunes gens qui rentraient d'Irak ou d'Afghanistan. Il sentait que les mêmes causes allaient produire les mêmes effets et qu'il y allait avoir un problème social dont personne n'avait idée $\mathrm{du}$ coût qu'il allait représenter. Et ces jeunes gens étaient pareils à leurs prédécesseurs du Vietnam, c'est-à-dire dans le déni. A part les cas les plus flagrants de PTSD, qui peuvent être décelés dans certains cas, tous disent en rentrant qu'il n'y a pas de problème. Ils ont envie de rentrer à la maison, de retrouver leurs femmes, leurs copines, leurs enfants, de reprendre une vie normale. Ils sont les premiers à être dans le déni. Ils n'ont surtout pas envie d'être stigmatisés.

GC : Ils ne veulent pas raconter leurs cauchemars.

LBR : Absolument. Ils n'ont pas envie d'avoir l'air d'être des lavettes. Ce qui veut dire que, du point de vue de Fred, et il avait raison, il faut être d'autant plus à l'affût, c'està-dire qu'il faut faire ce qu'on appelle "outreach ", c'est-à-dire aller les chercher, chez eux, systématiquement. D'autant que selon les études de la Rand Corporation commissionnées par le DOD, Département de la Défense, en 2009, un soldat sur trois rentre avec le PTSD. Donc il y a trois millions de jeunes Américains qui ont sont allés soit en Afghanistan, soit en Irak, ce qui veut dire qu'un million d'entre eux ont déjà PTSD en rentrant, ce qui ne préjuge pas du nombre qui l'auront dans cinq ans, dix ans, trente ans... Si on inclut les membres de la famille, ça fait plus de dix millions de personnes.

\section{GC : Comment s'est créé ce nouveau centre, où est tourné le film?}

LBR: Quand j'ai connu Fred en 2004, arrivaient chaque semaine dans son établissement de nouveaux patients, qui avaient entre 65 et 85 ans et qui n'avaient jamais parlé de la Seconde Guerre mondiale ou de la guerre du Vietnam. Et puis, ils avaient subi un nouveau traumatisme dans leur vie - la mort du conjoint, l'annonce d'une maladie grave, la perte d'un enfant - peu importe, pas pour eux, mais peu importe la nature du traumatisme. Tout à coup, ce n'était pas le traumatisme du moment qui importait, c'était la réactualisation du premier. Et ils cherchaient de l'aide.

Il est certain que les jeunes vétérans n'avaient pas envie d'aller dans ce type d'établissement, parce qu'ils n'avaient pas envie de se retrouver avec des patients qui avaient l'âge d'être leur grand-père. Les choses ont un peu changé depuis, mais c'était le cas a à l'époque. Donc en 2007, Fred a été contacté par un philanthrope de la Silicon Valley qui a proposé de mettre tant de millions de dollars pour créer ex nihilo un centre destiné à accueillir uniquement les vétérans des deux guerres, en Afghanistan et en Irak. Fred a conseillé d'embaucher plutôt un jeune pour s'en occuper mais ils n'ont trouvé personne et finalement, il a quitté l'établissement qu'il avait créé pour ceux du Vietnam pour s'occuper de ce nouveau centre. 
GC : On sent assez bien dans le film comment la caméra a pu contribuer à la thérapie dans le centre, mais le film comporte aussi des scènes en extérieur, qui créent d'ailleurs un effet libératoire. Vous suivez certains patients dans leurs familles, chez eux, dans des lieux publics. Comment le dialogue a-t-il pu se réamorcer dans ces circonstances ? Comment le fil s'est-il renoué?

LBR : La validation que ma présence pouvait apporter dans le contexte du travail thérapeutique, que j'ai évoquée plus haut, s'est en fait accentuée quand on s'est rendu dans les familles. Ce qu'on n'avait pas anticipé, pour le coup, c'est que dès qu'on arrivait - et parfois, on ne les avait pas vus depuis des mois, un an, voire plus ils reprenaient le fil là où ils l'avaient laissé dans la salle de thérapie, ce qui voulait dire à quel point ils nous avaient assimilés, sachant, et c'est très important, qu'ils n'ont jamais connu le centre de thérapie sans caméra. J'ai mis en place ce dispositif il y a vingt ans avec De guerre lasses, je ne l'ai jamais conçu autrement. Il était hors de question de débarquer dans quelque chose en cours. Soit on est à l'origine, et d'une certaine manière on fait partie du travail thérapeutique, soit je n'ai pas envie de le faire.

\section{GC : Donc il n'y a pas du tout d'interview?}

LBR : Jamais.

\section{GC : Aucune question directe?}

LBR : Non. Quand on est retourné les voir en famille, si, il y a un moment où on lance la conversation, mais ça roule tout seul parce que, encore une fois, ils nous ont assimilés à leur démarche. Et il se passe alors autre chose. Il se crée un triangle de paroles, sans doute entièrement inconscient. Que ce soit les garçons, les femmes, les enfants, les parents, tous utilisent la caméra pour se dire des choses qu'ils ne se disent pas dans le quotidien parce que dans le quotidien, il n'y a pas d'espace pour le dire. Donc l'arrivée de la caméra dans la maison crée un espace thérapeutique que chacun investit. Et c'est très touchant parce que là, quelque chose passe par la caméra, sans cabotinage, et sans qu'ils s'adressent à la caméra.

GC : La parole circule autrement.

LBR : C'est le cas notamment des deux petites filles, qui utilisent la caméra sans en avoir conscience, pour dire des choses à leur père.

GC : Celle qui fait ses devoirs avec son papa, qui justement, par moments, le regarde et fait comme si elle ne pouvait pas parler?

LBR : Oui, et que le père met au lit plus tard. Mais aussi la petite fille sur les épaules de son père dans la dernière scène. Je trouve ça extrêmement touchant parce que ça parle aussi du rôle qu'a joué ce film dans le travail thérapeutique et dans le cheminement des garçons et de leurs familles.

GC : Il y a dans votre film beaucoup de souffrance mais encore plus d'espérance. On en sort avec une certaine foi, dans la parole, dans la résistance, dans le projet lui-même.

LBR : Je suis très content que vous reconnaissiez ça, à plusieurs titres. D’abord parce que c'est mon point de départ, pour ce film, mais déjà il y a vingt ans. Parce que c'est de là qu'on vient, c'est-à-dire que les lignées auxquelles on appartient sont par essence des lignées survivantes, sinon on ne serait pas là. C'est donc qu'à un moment donné, face à des expériences extrêmement mortifères et celles du vingtième siècle le sont peut-être encore plus que toutes celles des siècles précédents, il y a eu quelque chose dans l'espèce de plus fort, vers la survivance. Après, tout le monde n'est pas égal dans ce domaine. Et les garçons, comme leurs devancières, les femmes dans $D e$ 
guerre lasses, sont des hommes et des femmes qui, en choisissant la parole, font un pas vers la survivance. C'est mon intime conviction. Si je choisis d'inscrire mon cinéma dans la thérapie, c'est aussi pour cette raison, parce que la parole est, dans un cas comme dans l'autre, un choix de la vie. Et ce n'est pas gagné. Elles luttent, ils luttent. Cela vient aussi du travail entrepris par le thérapeute qui a pour obsession de chercher un point d'ancrage vivant pour chacun. Où est-ce qu'ils pourront trouver assez de vivant en eux pour s'enraciner et reconstruire une nouvelle vie qui ne sera en rien celle qu'ils avaient avant, ou celle qu'ils avaient prévu d'avoir, mais la vie de celui qui a survécu à ce qui s'est passé ?

Alors évidemment peut-être que certains thérapeutes d'ici, qui viennent d'une culture plus essentiellement analytique, peuvent être surpris, mais il faut bien se remettre dans le contexte. On est face à de très grands traumatisés. Ce n'est qu'un premier travail, une toute première étape.

GC : Oui, enfin, c'est plus qu'une amorce.

LBR : Oui, mais il faudra continuer toute la vie. Ça ne sera jamais gagné. Je pense que l'espérance vient aussi de l'ambition du travail thérapeutique et au-delà des méthodes, je suis convaincu que c'est ce qui est important et ce à quoi on assiste.

Il se passe aussi un autre phénomène. C'est que quand on fait un film, on part d'une masse de matière colossale, trois cents heures pour le premier film en Bosnie, cinq cents pour celui-ci. Au-delà des chiffres, la matière est composée de beaucoup de mortifère. C'est normal, il y a beaucoup de choses qui tombent, qui sont dites en thérapie mais qui ont vocation à tomber. Le thérapeute cherche du vivant pour créer de l'ancrage, et évidemment, nous aussi, et même bien davantage, parce qu'on reformule en racontant une histoire. Ça ne m'intéresserait pas de restituer au public une histoire qui serait focalisée sur la mort parce que ce qui m'intéresse comme cinéaste, c'est ce qui est vivant. Justement, je veux mettre mes mains dans cette espèce de boue que sont ces cinq cents heures pour chercher du vivant. Et aussi pour le leur restituer parce que ce serait totalement criminel de ma part, sachant que je suis avec des personnes qui sont des vraies personnes avec des vrais traumatismes, des vraies questions et un vrai cheminement, de ne pas le restituer le vivant qui est en eux. Je ne l'envisagerais même pas.

GC : Oui. Est-ce qu'ils ont vu ce film? Est-ce que c'est nécessaire qu'ils le voient, à votre avis ? Ou est-ce que c'était le processus de thérapie qui était important?

LBR : Concrètement, ce qui était prévu avec Fred Gusman, c'est qu'on les réunisse tous et qu'ils passent un week-end avec leurs familles, leurs amis et qu'au cours de ce week-end, pour ceux qui veulent, on achève ce travail qu'on a fait ensemble. Ce qui devrait avoir lieu début 2015.

GC : II y a donc une suite prévue.

LBR : Il y a ce projet, mais il n'est pas sûr que tous en aient envie. Ils seront libres. Selon Fred, les garçons verront surtout la manière dont leur expérience est restituée sous forme d'un récit qui n'existe pas au jour le jour en thérapie, et qui fera sens pour eux dans la mesure où il peut être un éclairage pour tous leurs frères d'armes. Parce que, dans la culture militaire, cette question du frère d'armes revient constamment. Celui qu'on ne va pas laisser sur le champ de bataille et il y en a des centaines de milliers qui y sont encore. Ils ont sans doute ça en tête aussi. Je pense qu'il se passera beaucoup d'autres choses. Un des garçons l'a vu - celui qui se marie dans le film. Il se 
trouve que par sa femme, il a de la famille par alliance à Aix-en-Provence, et donc il est venu voir le film à Cannes [où le film était montré en sélection officielle hors compétition].

\section{GC : Il était au Festival ?}

LBR : Oui, il a monté les marches avec nous, il représentait un peu tous les autres. Trois jours après, je l'ai appelé au téléphone. Et il m'a dit qu'il s'était rendu compte à quel point il devait le chemin parcouru en thérapie au fait qu'ils l'avaient fait ensemble. Qu'à la fraternité d'armes s'est substituée une fraternité thérapeutique, et que c'est parce qu'ils ont baissé la garde tous ensemble qu'ils ont pu affronter chacun la fêlure profonde. Du coup, je crois qu'il en a éprouvé en voyant le film une très nostalgie vis-à-vis de ces frères de thérapie. Donc, en tout cas, il avait désir de nouveaux contacts avec eux. Parce qu'il mesurait en voyant le film combien d'être sous le regard de chacun d'entre eux lui avait apporté pour pouvoir lui-même faire face.

\section{GC : Etes-vous toujours un homme en colère?}

LBR : Ça se travaille. C'est aussi un travail de toute une vie, la colère. Et je pense surtout cette colère en héritage, dont je suis convaincu qu'elle est une grande partie du legs psychique de ces expériences traumatiques de guerre au vingtième siècle et que pour nos générations qui n'ont fait qu'hériter, elle est d'autant plus pénible qu'elle a encore moins d'objets. Ce n'est pas une colère contre ceci ou contre cela. C'est un état de colère. Chez eux aussi, c'est un état de colère.

GC : Ils sont la colère.

LBR : Ils sont la colère. Je pense qu'ils transmettent aussi la colère et qu'on on porte la colère. Effectivement, il y a quelque chose d'intuitif chez le cinéaste que je suis d'une perception de la colère qui est là, présente, pas uniquement en moi et autour de moi, mais dans la famille, mais au-delà dans la société à laquelle j'appartiens. Et une espèce de vision des contemporains comme des contemporains en colère. Et une interprétation, tout à fait subjective, que cette colère est l'héritage de ces expériences traumatiques de la guerre. En tout cas, je pense que les deux films apportent des réponses et permettent d'avoir presqu'une certaine tendresse vis-à-vis de cette colère et de dire "je sais d'où elle vient ", mais elle n'est pas nécessairement notre destin. L'affronter, comme je crois le faire à travers les deux films, peut me permettre à moi mais je pense aussi à d'autres de mes contemporains, de commencer à s'en affranchir.

GC : Merci à vous, et on attend donc le troisième et dernier volet de votre « généalogie de la colère ».

\section{AUTEUR}

\section{GÉRALDINE CHOUARD}

Université Paris-Dauphine 